\title{
Pyrimidine Derivatives as Promising Candidates for Potent Antiangiogenic: A silico Study
}

\author{
Nadhir N. A. Jafar ${ }^{1 *}$, Ahmed A. Hussein²
}

\author{
Pharmacy College, Al-Zahraa University for Women, Karbala 65001, Iraq. \\ 2Pharmacy Department, Al-Mustaqbal University College, 51001 Hillah, Babil, Iraq. \\ "Correspondence to: Nadhir N. A. Jafar (E-mail: nather.najim@alzahraa.edu.iq)
}

(Submitted:09 September 2021 - Revised version received: 22 September 2021 - Accepted: 01 October 2021 - Published online: 26 December 2021)

\begin{abstract}
Objectives: This study planned to explore the effect of many synthetic compounds derived from (4-chloro-6-methoxy- $\mathrm{N}, \mathrm{N}-\mathrm{dimethyl}$ pyrimidin-2-amine) as antiangiogenic.

Methods: Docking study has been done by using Molecular Operating Environment (2019) to examine the energy binding affinity of tested compounds with VEGFR-2 kinase and using Discovery Studio Visualizer v20.1.0.19295 free version to visualize the surface binding cavity.

Results: Theoretical calculation of these compounds showed significant results in comparing to the reference drug compounds, compound (1) which is 4-(4-(1,2,3-selenadiazol-4-yl)phenoxy)-6-methoxy-N,N-dimethylpyrimidin-2-amine gives the lowest binding energy equal to $(-8.116) \mathrm{Kcal} / \mathrm{mol}$ and nearest to the reference drug compound, and also it has excellent RMSD equal to (0.9263). The other compounds 4-(4-(1,2,3-thiadiazol-4-yl) phenoxy)-6-methoxy-N,N-dimethylpyrimidin-2-amine, 4-methoxy-N,N-dimethyl-6-(phenylthio) pyrimidin-2amine, 4-(benzo[d] thiazol-2-ylthio)-6-methoxy- $\mathrm{N}, \mathrm{N}$-dimethylpyrimidin-2-amine have $-7.739,-7.211$ and $-7.841 \mathrm{Kcal} / \mathrm{mol}$ binding energy and 2.668, 1.745 and 1.377 RMSD respectively.

Conclusion: Compound (1) can be recommended as a powerful antiangiogenic due to its theoretical results for binding energy.

Keywords: 1,2,3-seleniadiazole, anti-angiogenesis, anticancer, binding energy, pyrimidine
\end{abstract}

\section{Introduction}

Cancer cells have a very high metabolism process in comparison with normal cells and this led to high oxygen demand and more supplying of nutrients, this issue causes hypoxia which is considered the main regulation mechanism for angiogenesis. ${ }^{1,2}$ Scientists have been researched and concentrated on how tumors get the oxygen and nutrients needed. In 1989, the vascular endothelial growth factor (VEGF) protein was discovered by a team of scientists due to the belief that it induces angiogenesis ${ }^{3}$ by binding to their receptors in the endothelial cells of blood vessels and stimulate proliferation. ${ }^{4-6}$

The induction from the tumor itself causing angiogenesis, vasculogenesis, increasing vessel permeability, and migration to be around the tumor to supply it with oxygen and nutrients. ${ }^{7,8}$ The VEGF receptor inhibitors play an important role in the treatment of tumors by causing tumor starvation by preventing the proliferation of endothelial blood vessels that led to decreasing of oxygen and nutrients they need ${ }^{9}$ and will give tumor growth suppression ${ }^{10}$ such as Sorafenib (Nexavar) which has been approved as a potent antiangiogenic drug. ${ }^{11}$ The first objective of this study is testing in-silico pyrimidine derivative as a new pharmacophore antiangiogenic ligand, it is safer because it is a part unit in nucleic bases of RNA and DNA and this feature gave its derivatives widespread pharmacological activities. ${ }^{12,13}$ Pyrimidine derivatives have been used as potent anticancer in cases of breast, stomach, colon, pancreatic, and other types of cancer, ${ }^{14,15}$ antimicrobial effects, ${ }^{14,16,17}$ potent antitubercular agents, ${ }^{18}$ antimalarial effects especially for resistant species, ${ }^{19}$ potent anti-inflammatory, ${ }^{20}$ efficient antiviral activity, antihypertensive activity ${ }^{21}$ and especially for Varicella-zoster virus. ${ }^{22,23}$ The second objective of our study is to figure out a new activity of previously synthesized compounds with pyrimidine ring such as [1,2,3-selenadiazole, 1,2,3-thiadiazole, benzo[d]thiazol-2-ylthio] and thiophenyl compounds. $^{24}$

These compounds have been studied are organosulfur and organoselenium compounds which have significant activity as anti-fungal activity, ${ }^{24}$ antibacterial effect, ${ }^{25-30}$ due to the patients with cancer that suffering from microbial infections, ${ }^{31}$ so the most significant features will be emphasized in this article for pyrimidine with the substitutions that mentioned using a potent anticancer and antimicrobial activities to produce a single drug with double activities to add a new cost-effective feature. Consequently, merged the pyrimidine with fourth substitutions to discover in silico a new activity and to get more potent binding energy with vascular endothelial growth factor receptor protein.

\section{Materials and Methods \\ Docking Requirements}

Docking study has been done by using Molecular Operating Environment $(2019)^{32}$ to examine the energy binding affinity of tested compounds below with VEGFR-2 kinase and using Discovery Studio Visualizer v20.1.0.19295 free version ${ }^{33}$ to visualize the surface binding cavity.

\section{Preparation of Ligands}

The compounds that have been used were already synthesized, then, they have been processed by MOE in form of $2 \mathrm{D}$, prepared with protonate $3 \mathrm{D}$, partial charge automatically calculated, energy minimization to get the most stable conformation and saved in form 3D form as (moe) file, then all of them have been imported in database and saved as MDB file to be used in docking process. ${ }^{34,35}$ 


\section{Protein Arrangement and Preparation}

The crystal structure of the VEGFR2 kinase which is complex with PF- 00337210 (N,2-dimethyl-6-(7-(2-morpholinoethoxy) quinoline-4-yloxy)benzofuran-3-carboxamide) that has been downloaded from the Protein Data Bank website (PDB ID code is: 2XIR) with resolution: $1.50 \AA .{ }^{36}$ Preparation of crystal structure of a protein by adding hydrogen atoms during protonation 3D has been done at first, then checking all atom's connection errors have been done by automatic correction, potential fixation of all protein atoms with Amber12: EHT. Finally, Selection of all active sites and the creation of them as dummies by using a site finder (Figure 1)..$^{34,35}$

\section{Results and Discussion}

The results are given in Table 1, which explains the core compound which gives good interaction with low binding energy equal to $(-5.911)$ with RMSD equal to $(0.691)$, whereas the substituted synthesized compounds $1,2,3$, and 4 gave the lowest binding energy equal to $(-8.116,-7.739,-7.211$ and $-7.84)$, respectively, and nearest to the reference drug compound, and also it has very good RMSD equal to (0.9263), that means substations play an important role in the protein binding process in comparing with the starting pyrimidine derivative compound (5).

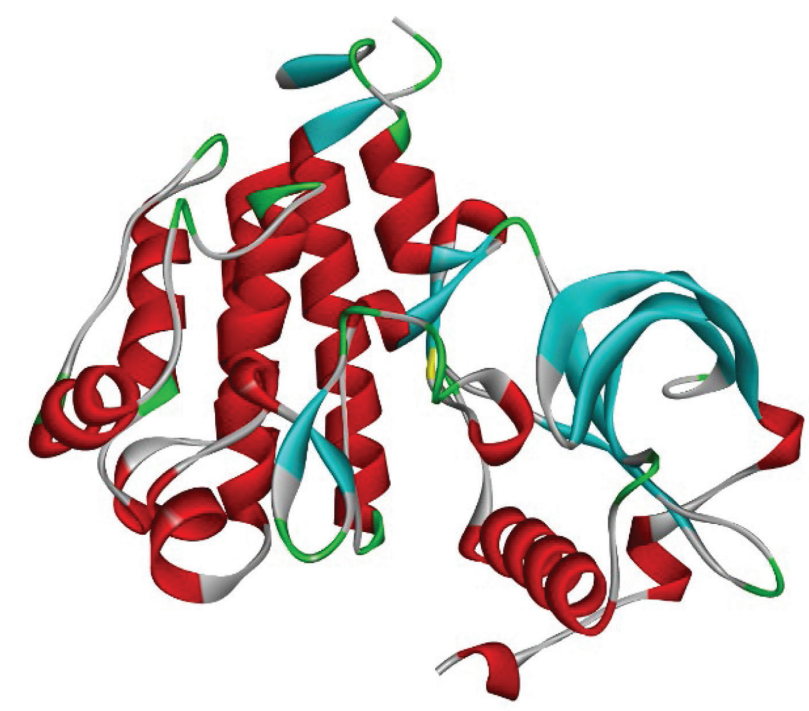

Fig. 1 Crystal structure of the VEGFR2 kinase PDB code (2XIR).

\section{Docking Study}

The prepared database of compounds mentioned previously has been tested on the protein VEGA-2 receptor. Generally, the docking was done by loading the protein with dummies atoms that represent active sites in the protein, modifying the default properties in MOE software, set docking site as dummy atoms, ligand as $\mathrm{MDB}$ file, rigid receptor method, triangle matcher as method placement, London dG and GBVI/WSA $\mathrm{dG}$ as score method and have been selected the best 10 confirmations out of 200 different conformations for each ligand. Then we chose the best pose that has the lowest energy with good RMSD values from the obtained docking results. . $5,37,38^{-1}$

The compound (1) has been interacted with protein amino acids [CYS 919 (A) H-acceptor interaction of (N) with (N25) in ligand; ASP 1046 (A) H-acceptor interaction of (N) with (N27) in ligand; LEU 840 (A) pi-H interaction of (CD1) with (5-ring) in ligand; VAL 899 (A) pi-H interaction of (CG1) with (6-ring) in ligand] with distances $3.14 \AA$, $3.21 \AA$, $3.82 \AA$, $4.44 \AA$ and $\mathrm{E}(\mathrm{kcal} / \mathrm{mol})-0.7,-2.4,-0.6,-0.6$ respectively as shown in Figures 2 and 3.

The compound (2) has interacted with amino acids [ASP 1046 (A) $\mathrm{H}$-acceptor interaction of $(\mathrm{N})$ with $(\mathrm{O})$ in ligand; LEU 840(A) pi-H interaction of (CD2) with (5-ring) in ligand; LYS 868(A) pi-H interaction of (CE) with (6-ring) in ligand] with distances $2.95 \AA, 3.95 \AA, 4.27 \AA$ and $\mathrm{E}$ (kcal/mol) -1.8, $-1.2,-1.2$ respectively. The compound (3) has interacted with amino acids [ASP 1046 (A) H-acceptor interaction of (N) with (O) in ligand; VAL 848(A) pi-H interaction of (CG1) with (6-ring)

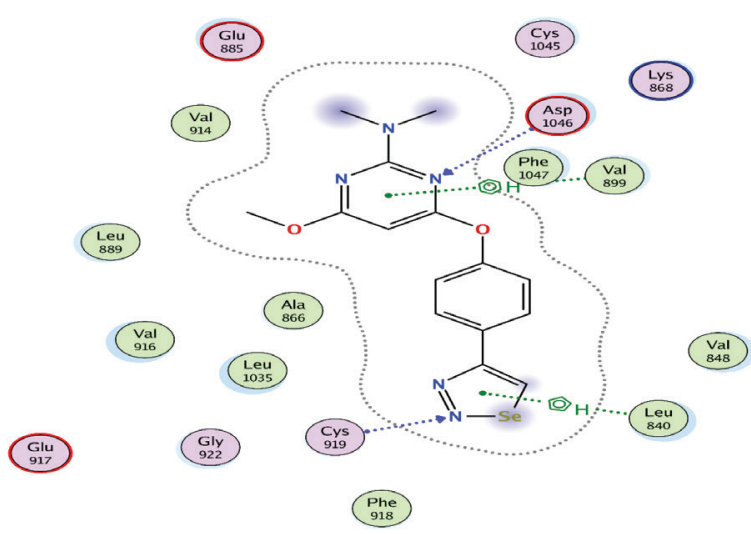

Fig. 2 Diagram interaction of compound (1) with the crystal structure of the VEGFR2 kinase.

\begin{tabular}{ccccccccc}
\hline \multicolumn{2}{l}{ Table 1. Binding energies of the ligand with protein } \\
\hline No. & Compound & S & rmsd_refine & E_conf & E_place & E_score1 & E_refine & E_score2 \\
\hline 1 & Reference & -9.822 & 1.516 & 11.596 & -67.448 & -16.645 & -45.713 & -9.822 \\
2 & 1 & -8.116 & 0.926 & -100.626 & -55.464 & -9.436 & -36.111 & -8.116 \\
3 & 2 & -7.739 & 2.668 & -89.229 & -56.335 & -9.539 & -33.926 & -7.739 \\
4 & 3 & -7.211 & 1.745 & -118.107 & -61.455 & -9.759 & -31.147 & -7.211 \\
5 & 4 & -7.841 & 1.377 & -107.456 & -55.826 & -9.683 & -36.329 & -7.841 \\
6 & 5 & -5.911 & 0.691 & -128.838 & -56.396 & -8.053 & -29.731 & -5.911 \\
\hline
\end{tabular}

S, The final score; RMSD, Root-mean-square deviation between the pose before refinement and the pose after refinement; E_conf, The energy of the conformer; E_place, Score from the placement stage; E_score1, Score from the rescoring stage(s); E_refine, Score from the refinement stage and No. of conf-number of conformations generated by ligand. 
in ligand; LYS 868(A) pi-H interaction of (CE) with (6-ring) in ligand] with distances $2.94 \AA, 4.42 \AA, 4.07 \AA$ and $\mathrm{E}(\mathrm{kcal} / \mathrm{mol})$ $-1.9,-0.7,-1.1$ respectively. The compound (4) has interacted with amino acids [ASP 1046 (A) H-acceptor interaction of (N) with (N9) in ligand; VAL 848(A) pi-H interaction of (CG1) with (5-ring) in ligand] with distances $3.26 \AA, 4.23 \AA$ and $\mathrm{E}$ (kcal/mol) $-1.6,-0.9$ respectively.

\section{Structure-Activity Relationship (SAR)}

The primary study of SAR has focused on the impact of substituents in replacing chlorine atoms in the pyrimidine core, which is related to $\mathrm{H}$-bond accepter via $\mathrm{N}$ or $\mathrm{O}$-atoms or arene amino acid interaction.

The compound (5) represents a core compound that has been used to synthesize other four compounds which have been interacted with amino acids [ASP 1046 (A) H-acceptor interaction of $(\mathrm{N})$ with (N2) in ligand; VAL 899(A) pi-H interaction of (CG1) with (6-ring) in ligand] with distances $3.5 \AA$, $4.43 \AA$ and $\mathrm{E}(\mathrm{kcal} / \mathrm{mol})-1.2,-0.6$ respectively as shown in Figure 4 . When replacing chloride in the core compound with four different substituents separately as shown in Scheme 1. Compound 1 has been exhibited $-8.116 \mathrm{Kcal} / \mathrm{mol}$ binding energy because increasing the hydrogen bonds accepter -4.100 $\mathrm{Kcal} / \mathrm{mole}$ related with $\mathrm{N}$ interacted with ASP 1046 (A) compared with the core compound equal to $-1.200 \mathrm{Kcal} / \mathrm{mol}$. Furthermore, the presence of $-1.600 \mathrm{Kcal} / \mathrm{mole}$ hydrogen acceptor

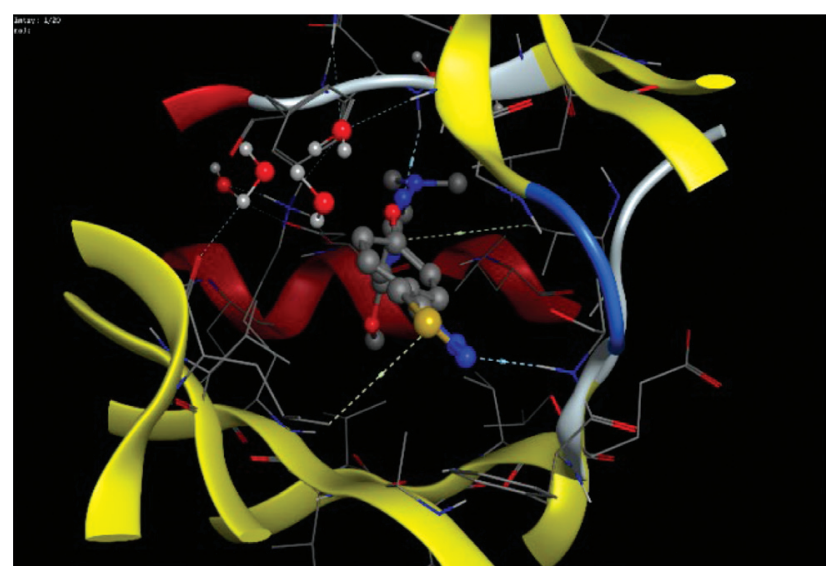

Fig. 3 Diagram isolated interaction of compound (1) with the crystal structure of the VEGFR2 kinase.

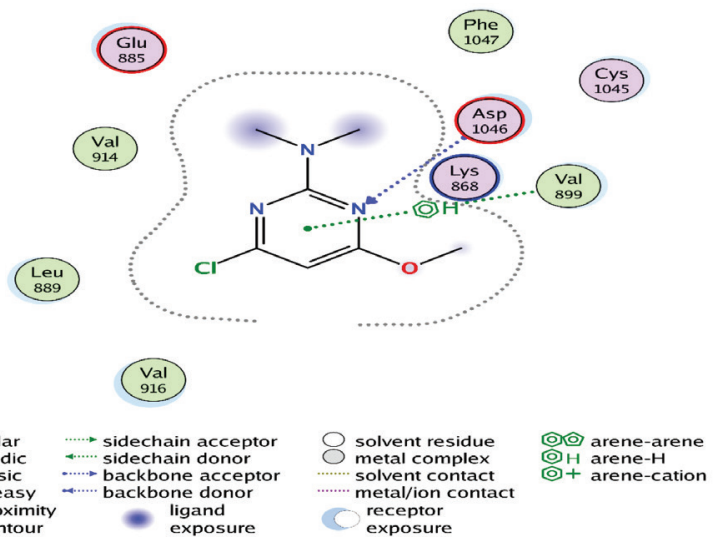

Fig. 4 Diagram interaction of compound (5) with the crystal structure of the VEGFR-2. of $\mathrm{N}$ with CYS 919 (A) and changing the interaction of third connect with LYS 868(A) instead of VAL 899 (A) that means the compound has been orientated with the best alignment with protein as in Figures 2, 3.

Compound 2 has appeared $-7.739 \mathrm{Kcal} /$ mole binding energy from $\mathrm{H}$-acceptor O with ASP 1046 (A) and two Pi-H interactions as in Figure 5, compound 3 and 4 have -7.211 and $-7.841 \mathrm{Kcal} / \mathrm{mol}$, respectively, as could be observed in Figures 6 and 7.

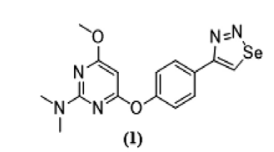
4-(4-(1,2,3-selenadiazol-4-yl)phenoxy)-6-
methoxy- $N, N$-dimethylpyrimidin-2-amine methoxy- $N, N$-dimethylpyrimidin-2-amine
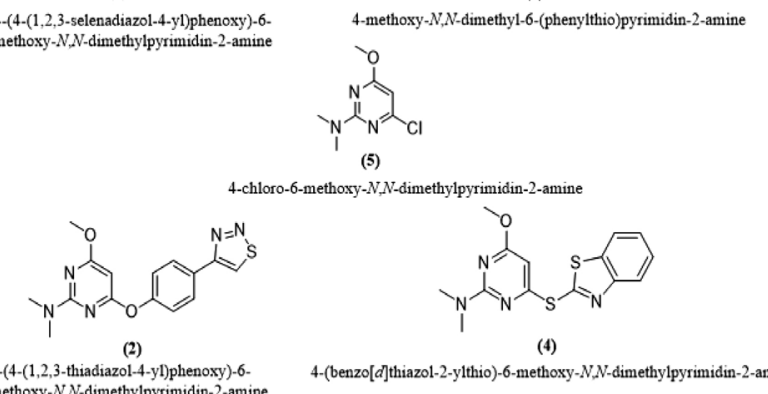
4(4-(1,2,3-thadiazol-4-yl)phenoxy)-6-

Scheme 1. Structures of Pyrimidine and four synthesized compounds.

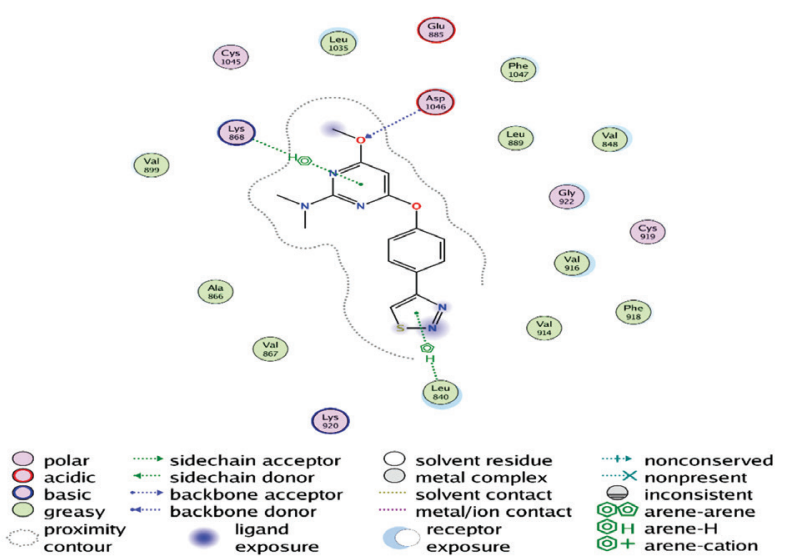

Fig. 5 Diagram interaction of compound (2) with the crystal structure of the VEGFR-2.

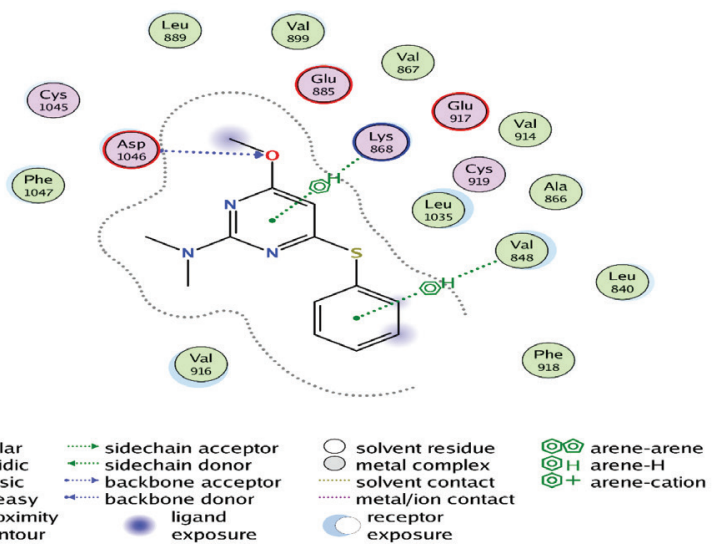

Fig. 6 Diagram interaction of compound (3) with the crystal structure of the VEGFR-2. 


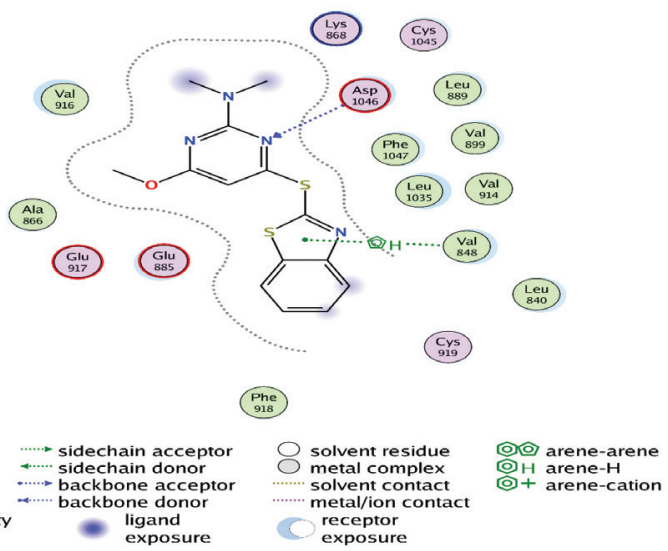

Fig. 7 Diagram interaction of compound (4) with the crystal structure of the VEGFR-2.

The theoretical results of those compounds have been similar, approximately, to the result of reference drugs (Sorafenib) and (Axitinib) which have binding energy equal to
-7.4 and -8.4 , respectively, this issue encourages us to proceed for furthermore studies.

\section{Conclusion}

This research contributes to finding a new core from pyrimidine derivatives as promising candidates for powerful antiangiogenic drug in-silico study. Further investigations are recommended for new researchers as driving the possibility for more broad pharmacological examinations.

The docking studies and flexible alignment would propose a good affinity of most of the synthesized compounds towards interacting with VEGFR-2 receptor in better binding energy from the data obtained, all compounds exhibited $-8.116,-7.739,-7.211$ and $-7.841 \mathrm{Kcal} / \mathrm{mol}$ binding energy, respectively, with compounds 1, 2, 3 and 4 with small RMSD.

The results demonstrated that the most dynamic mixes could be helpful as a format for future patterns, advancement, modification, furthermore, assessment to make more extraordinary and specific VEGFR-2 inhibitors with higher anticancer analogs.

\section{Conflict of Interest: None.}

\section{References}

1. Garcia J, Hurwitz HI, Sandler AB, et al. Bevacizumab (Avastin ${ }^{\oplus}$ ) in cancer treatment: A review of 15 years of clinical experience and future outlook. Cancer Treat Rev 2020; 86: 102017.

2. Tanimoto $\mathrm{K}$, Yoshiga $\mathrm{K}$, Equchi $\mathrm{H}$, et al. Hypoxia-inducible factor-1a polymorphisms associated with enhanced transactivation capacity, implying clinical significance. Carcinogenesis 2003; 24: 1779-1783.

3. Tischer E, Gospodarowicz D, Mitchell R, et al. Vascular endothelial growth factor: A new member of the platelet-derived growth factor gene family. Biochem Biophys Res Commun 1989; 165: 1198-1206.

4. Ferrara N, Henzel WJ. Pituitary follicular cells secrete a novel heparin-binding growth factor specific for vascular endothelial cells. Biochem Biophys Res Commun 1989; 161: 851-858.

5. Connolly DT, Heuvelman DM, Nelson R, et al. Tumor vascular permeability factor stimulates endothelial cell growth and angiogenesis. J Clin Invest 1989; 84: 1470-1478.

6. Leung DW, Cachianes G, Kuang W-J, et al. Vascular endothelial growth factor is a secreted angiogenic mitogen. Science (80-) 1989; 246: 1306-1309.

7. Hicklin DJ, Ellis LM. Role of the vascular endothelial growth factor pathway in tumor growth and angiogenesis. J Clin Oncol 2005; 23: 1011-1027.

8. Lugano R, Ramachandran M, Dimberg A. Tumor angiogenesis: causes, consequences, challenges and opportunities. Cell Mol Life Sci 2020; 77: $1745-1770$.

9. Jain RK. Antiangiogenesis Strategies Revisited: From Starving Tumors to Alleviating Hypoxia. Cancer Cell 2014; 26: 605-622.

10. Ferrara N, Hillan KJ, Gerber H-P, et al. Discovery and development of bevacizumab, an anti-VEGF antibody for treating cancer. Nat Rev Drug Discov 2004; 3: 391-400.

11. Wu P, Nielsen TE, Clausen MH. FDA-approved small-molecule kinase inhibitors. Trends Pharmacol Sci 2015; 36: 422-439.

12. Fargualy AM, Habib NS, Ismail KA, et al. Synthesis, biological evaluation and molecular docking studies of some pyrimidine derivatives. Eur J Med Chem 2013; 66: 276-295.

13. Sharma V, Chitranshi N, Agarwal AK. Significance and biological importance of pyrimidine in the microbial world. Int J Med Chem 2014 2014: 202784

14. Habib NS, Soliman R, Ismail K, et al. Pyrimidines. Part II: Synthesis of novel pyrimidines, 1,2,4-triazolo[4,3-a]pyrimidin-7-ones and pyrimidino[2,1-c] $[1,2,4]$ triazin-8-ones for their antimicrobial and anticancer activities. Boll Chim Farm 2003; 142: 396-405.

15. Kumar S, Deep A, Narasimhan B. A review on synthesis, anticancer and antiviral potentials of pyrimidine derivatives. Curr Bioact Compd 2019; 15: 289-303.

16. Ram VJ, Berghe DAV, Vlietinck AJ. 5-Cyano-6-aryluracil and 2-thiouracil derivatives as potential chemotherapeutic agents. IV. Journal of Heterocyclic Chemistry 1984; 21: 1307-1312.

17. Mallikarjunaswamy C. Studies on synthesis of pyrimidine derivatives and their antimicrobial activity. Arab J Chem 2017; 10: S484-\$490.

18. Liu P, Yang Y, Tang Y, et al. Design and synthesis of novel pyrimidine derivatives as potent antitubercular agents. Eur J Med Chem 2019; 163: 169-182.

19. Singh K, Kaur T. Pyrimidine-based antimalarials: Design strategies and antiplasmodial effects. Medchemcomm 2016; 7: 749-768.

20. Naik NS, Shastri LA, Chougala BM, et al. Synthesis of novel aryl and coumarin substituted pyrazolo[1,5-a]pyrimidine derivatives as potent antiinflammatory and anticancer agents. Chem Data Collect 2020; 30: 100550

21. Rani J, Kumar S, Saini M, et al. Biological potential of pyrimidine derivatives in a new era. Res Chem Intermed 2016; 42: 6777-6804.

22. McGuigan C, Barucki H, Blewett S, et al. Highly potent and selective inhibition of varicella-zoster virus by bicyclic furopyrimidine nucleosides bearing an aryl side chain. J Med Chem 2000; 43: 4993-4997.

23. Luo M, Groaz E, Snoeck R, et al. Amidate Prodrugs of O-2-Alkylated Pyrimidine Acyclic Nucleosides Display Potent Anti-Herpesvirus Activity. ACS Med Chem Lett 2020; 11: 1410-1415.

24. Jafar NNA, Mahdi IMA, Hadwan MH, et al. The antifungal effect of some 4-chloro-6-methoxy-N, N-dimethylpyrimidin-2-amine derivatives containing a heterocyclic compound on the important types of fungi. J Young Pharm 2017; 9: 463-467.

25. Lalezari I, Shafiee A, Yazdany S. Selenium heterocycles X: Synthesis and antibacterial activity of pyridyl-1,2,3-thiadiazoles and pyridyl-1,2,3selenadiazoles. J Pharm Sci 1974; 63: 628-629.

26. Al-Smadi M, Al-Momani F. Synthesis, characterization and antimicrobial activity of new 1,2,3-selenadiazoles. Molecules 2008; 13: 2740-2749.

27. Karnik A.V., Kulkarni AM, Malviya NJ, et al. Synthesis and in vitro antibacterial evaluation of tetracyclic-ortho-fused 4H-naphtho[1',2'-5,6] pyrano[3,4-d](1,2,3)selenadiazole and its derivatives. Eur J Med Chem 2008; 43: 2615-2617.

28. Gawad J, Bonde C. Design, synthesis and biological evaluation of some 2-(6-nitrobenzo[d]thiazol-2-ylthio)-N-benzyl-N-(6-nitrobenzo[d]thiazol-2yl)acetamide derivatives as selective DprE1 inhibitors. Synth Commun 2019; 49: $2696-2708$

29. Rajendran N, Kamatchi N, Periyasamy A, et al. DNA-interaction, antibacterial and in vitro cytotoxic properties of copper(II) complexes bearing (E)-2-(2(benzo[d]thiazol-2-ylthio)-1-phenylethylidene)thiosemicarbazone and diimine co-ligands. J Coord Chem 2020; 73: 969-985.

30. Gularte MS, Anghinoni JM, Abenante L, et al. Synthesis of chitosan derivatives with organoselenium and organosulfur compounds: Characterization, antimicrobial properties and application as biomaterials. Carbohydr Polym 2019; 219: 240-250.

31. Singer C, Kaplan MH, Armstrong D. Bacteremia and fungemia complicating neoplastic disease. A study of 364 Cases. Am J Med 1977; 62: 731-742. 
32. Inc. CCG. Molecular operating environment (MOE).

33. Biovia DS. BIOVIA workbook, release 2017; BIOVIA pipeline pilot, release 2017. San Diego Dassault Systèmes.

34. Ghanem A, Emara HA, Muawia S, et al. Tanshinone IIA synergistically enhances the antitumor activity of doxorubicin by interfering with the $\mathrm{PI3K/AKT/mTOR} \mathrm{pathway} \mathrm{and} \mathrm{inhibition} \mathrm{of} \mathrm{topoisomerase} \mathrm{II:} \mathrm{In} \mathrm{vitro} \mathrm{and}$ molecular docking studies. New J Chem 2020; 44: 17374-17381.

35. Zaki AA, Al-Karmalawy AA, El-Amier YA, et al. Molecular docking reveals the potential of Cleome amblyocarpa isolated compounds to inhibit COVID-19 virus main protease. New J Chem 2020; 44: 16752-16758.
36. Marrone T, Hu-Lowe D, Grazzini M, et al. PF-00337210, a potent, selective and orally bioavailable small molecule inhibitor of VEGFR-2.

37. Ghanem A, Emara HA, Muawia S, et al. Tanshinone IIA synergistically enhances the antitumor activity of doxorubicin by interfering with the PI3K/AKT/mTOR pathway and inhibition of topoisomerase II: in vitro and molecular docking studies. New J Chem.

38. Amin LHT, Shawer TZ, El-Naggar AM, et al. Design, synthesis, anticancer evaluation and docking studies of new pyrimidine derivatives as potent thymidylate synthase inhibitors. Bioorg Chem 2019; 91: 103159

This work is licensed under a Creative Commons Attribution-NonCommercial 3.0 Unported License which allows users to read, copy, distribute and make derivative works for non-commercial purposes from the material, as long as the author of the original work is cited properly. 